\title{
Vibration protection of sensitive components of infrared equipment in harsh environments
}

\author{
A.M. Veprik ${ }^{\mathrm{a}, *}$, V.I. Babitsky ${ }^{\mathrm{a}}$, N. Pundak ${ }^{\mathrm{b}}$ and \\ S.V. Riabzev ${ }^{\text {b }}$ \\ ${ }^{a}$ Department of Mechanical Engineering, \\ Loughborough University, Loughborough, \\ Leicestershire, LE11 3TU, UK \\ ${ }^{\mathrm{b}} R \& D$ Department, RICOR, Cryogenic and Vacuum \\ Systems, Kibbutz En Harod Ihud, 18960, Israel
}

This article addresses the principles of optimal vibration protection of the internal sensitive components of infrared equipment from harsh environmental vibration. The authors have developed an approach to the design of external vibration isolators with properties to minimise the vibration-induced line-of-sight jitter which is caused by the relative deflection of the infrared sensor and the optic system, subject to strict constraints on the allowable sway space of the entire electrooptic package. In this approach, the package itself is used as the first-level vibration isolation stage relative to the internal highly responsive components.

It was predicted analytically, and confirmed experimentally, that the proposed vibration isolation system would be capable of a sixfold reduction of the dynamic response of the infrared sensor as compared to the case of rigid mounting of the entire package.

\section{Introduction}

Infrared (IR) imagers enhance tremendously the ability to detect and track ground, sea and air targets, and also to navigate at nighttime $[2,6]$. Their operating principle is based on that simple fact that warmer objects radiate more and cooler objects radiate less. Since their noise figure strongly depends on the operating temperature of the IR detector, a high-resolution imager requires cryogenic cooling down to $80 \mathrm{~K}$ and a high level of optic stabilisation.

Modern sophisticated airborne thermal imagers, which require compact design, low input power and

*Corresponding author. Tel.: +44 1509 223177; Fax: +44 1509 223934; E-mail: A.Veprik@lboro.ac.uk. long life-times, often rely on closed cycle cryogenic coolers. Stirling coolers are especially suitable for such applications. Compared to Gifford-McMahon and Joule-Thomson cycles, Stirling offers more than twice the cooling performance in the cooling power range 1$100 \mathrm{~W}$. The application of new technologies allows the life-time figures for Stirling coolers to be well beyond 40000 hours [5].

Stirling coolers, which may be of both split and integral types [6,11], typically comprise two major components: a compressor and an expander. In a split cooler these are interconnected by a flexible gas transfer line (a thin-walled stainless steel tube of a small diameter) to provide for maximum flexibility in the system design and to isolate the IR detector from the vibration interference which is produced by the compressor. In the integral cooler these components are integrated in a common casing.

The reciprocating motion of a compressor piston provides the required pressure pulses and the volumetric reciprocal change of a working agent (helium, typically) in the expansion space of an expander. A displacer, which is located inside a cold finger, shuttles the working agent back and forth from the cold side to the warm side of the cooler. During the expansion stage of the thermodynamic cycle, heat is absorbed from the cold finger tip (cold side of a cycle), and during the compression stage, heat is rejected to the ambient from the cold finger base (warm side of a cycle) $[6,11]$.

It is a modern tendency to mount the IR sensor directly upon the cold finger tip. Such a concept, which is known as Integrated Dewar Cooler Assembly (IDCA), allows for practical elimination of the integration losses and better temperature uniformity across the IR sensor, as compared, for example, with the old-fashioned sleep-on design [6].

The typical layout of an IDCA, which relies on the integral Stirling cryocooler RICOR model K508A, is shown in Fig. 1.

Figure 2 shows the schematics of the integrated electro-optic package containing the integral cryogenic cooler (1) which carries the IR sensor (2) upon cold fin- 


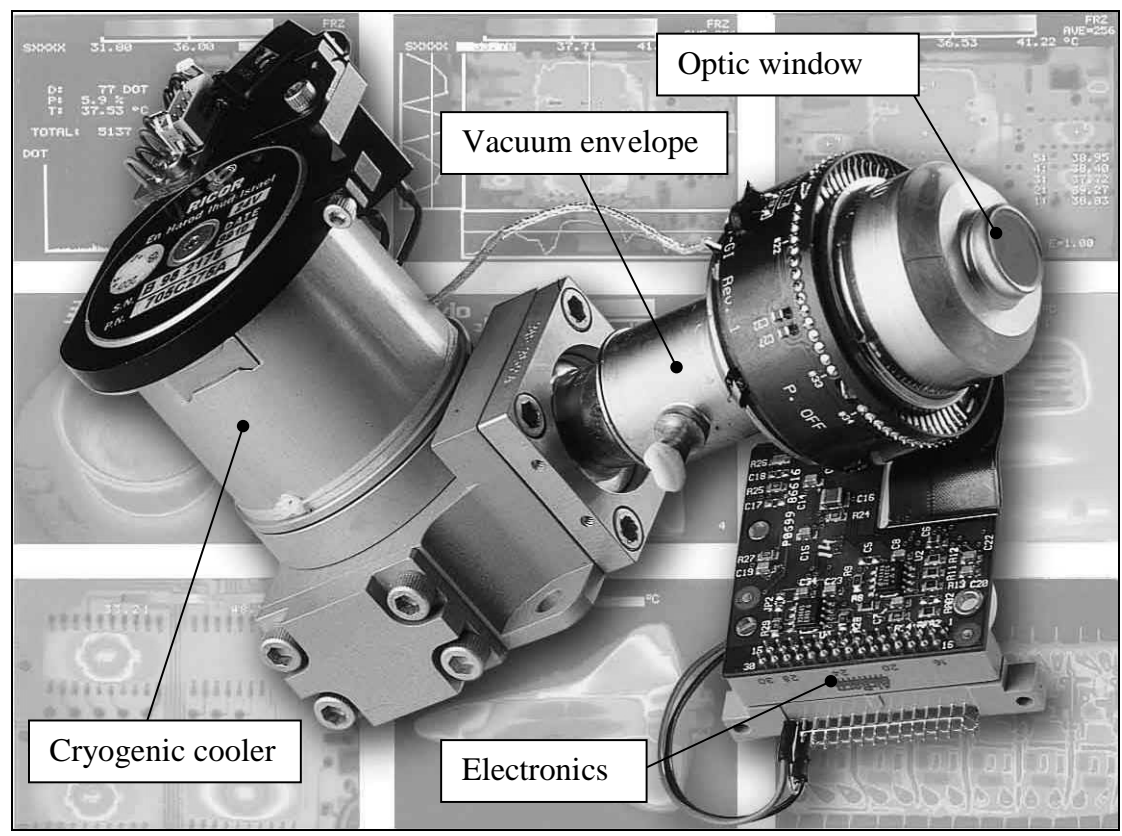

Fig. 1. Typical layout of IDCA which relies on integral Stirling cryocooler RICOR model K508A.

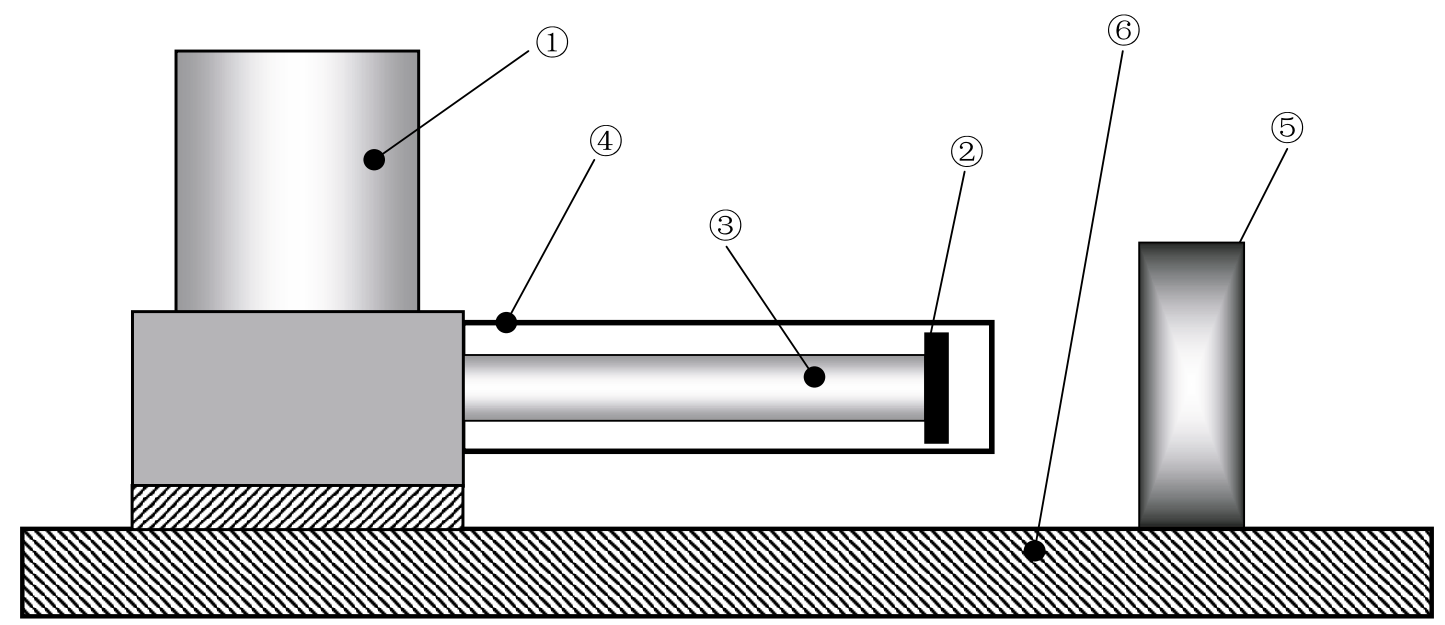

Fig. 2. Schematics of integrated electro-optic package.

ger tip (3). Both cold finger and IR sensor are located inside the vacuum dewar envelope (4). The cryogenic cooler, along with the appropriate optics (5), is mounted upon the single rigid structure (optic bench) which is required for proper optic alignment and stabilisation and also for placement of accompanying electronics.

Figure 3 shows a typical electro-optic device which relies on the integral RICOR model K508A cryogenic cooler.

The well-known drawback of the electro-optic devices which rely on the IDCA concept is their high sen- sitivity to external broadband random vibration. This is because, since it is desirable to decrease the heat conductivity of the cold fingers, they are typically thinwalled and manufactured of low-conductive alloys such as stainless steel or titanium. A cold finger which carries an IR sensor may be treated as a cantilever beam with an end lump mass. As a result of the low stiffness and damping intrinsic in such a structure, the IR sensor behaves as a lightly damped dynamic system the principal natural frequency of which falls into the frequency range of external disturbances. Wideband 


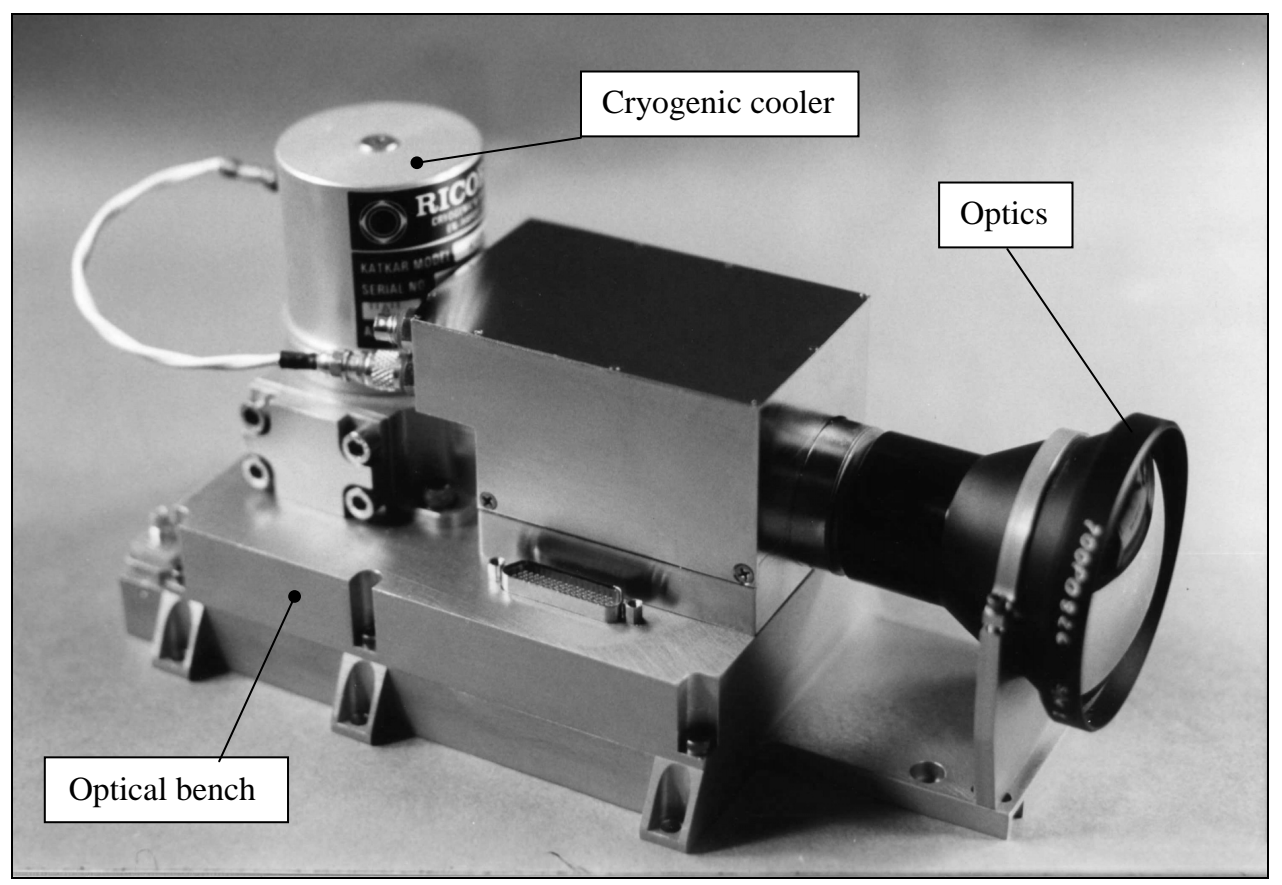

Fig. 3. Typical electro-optic device.

random excitation, therefore, may give rise to a quasiresonant dynamic response of the sensor relative to the rest of the optic system. As the level of line-of-sight jitter becomes comparable with the spatial resolution of a particular sensor, the vibration components contaminate the video signal causing the drastic degradation in performance of the IR imager.

Figure 4 shows the experimentally measured universal relative transmissibility of the IR sensor in a typical IDCA design. From curve-fitting based identification, the natural frequency is $816 \mathrm{~Hz}$ and the loss factor is $3.2 \%$. Such a low loss factor and natural frequency explains high vulnerability of the IDCA design to external random wideband vibration, the excitation spectrum of which usually contains essential frequency components up to $2000 \mathrm{~Hz}$.

The known approaches to ruggedizing of vibration sensitive components involve different combinations of stiffening and damping treatments. These methods aim to either reduce the resonant amplitudes by damping, or avoid them altogether by increasing the relevant resonant frequencies to above excitation frequency.

However, the traditional methods of ruggedizing become inadequate for the vibration control of the cold finger of a cryogenic cooler. An increase in the wall thickness of a cold finger and application of additional supports for extra stiffness lead to an excessive growth in heat loading through the increase in conductivity and shuttle losses. Since outgassing inside the high vacuum envelope is a concern, the application of the typical polymer materials for damping becomes practically impossible.

To combat the problem of excessive response of the IR sensor the designers are now looking at using vibration isolators. As the static and dynamic alignment of the IR sensor relative to the rest of the optical system is a concern, the vibration isolation of the entire electrooptic package is the only option. It is important to note now that large dynamic response of the entire isolated package involves "solid body" modes of motion and takes place at typically low frequencies. Since the deflection of the IR sensor relative to the optic system is not involved in such motion, the quality of the IR imaging of the remote targets is not affected. Figure 5 shows the schematics of such an isolated IR device.

Vibration isolation is the simplest, most widespread and well-studied method of vibration protection [4,7]. As is known, the best isolation in the typical high frequency span may be achieved when the natural frequency and loss factor of the vibration isolator are low. Unfortunately, such an isolator is only feasible in such applications where the intensive shock, random excitation and constant accelerations (g-loads) are not typical. 


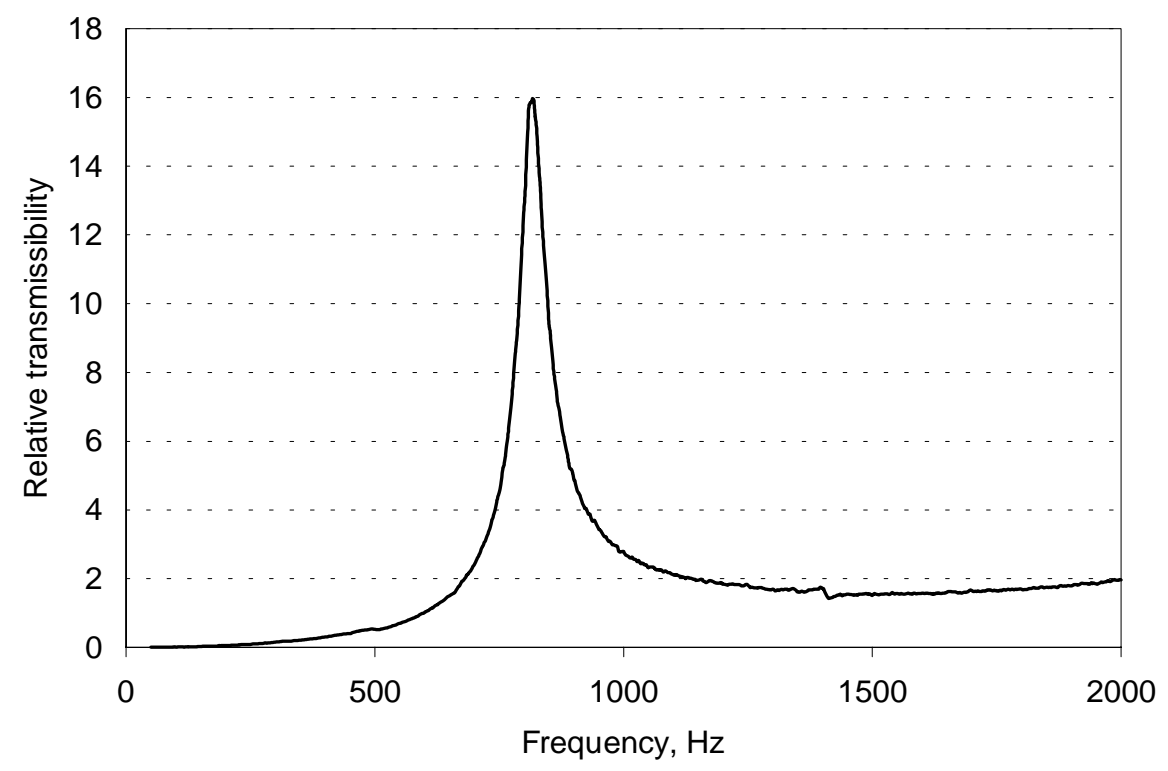

Fig. 4. Experimentally measured absolute transmissibility of IR sensor.

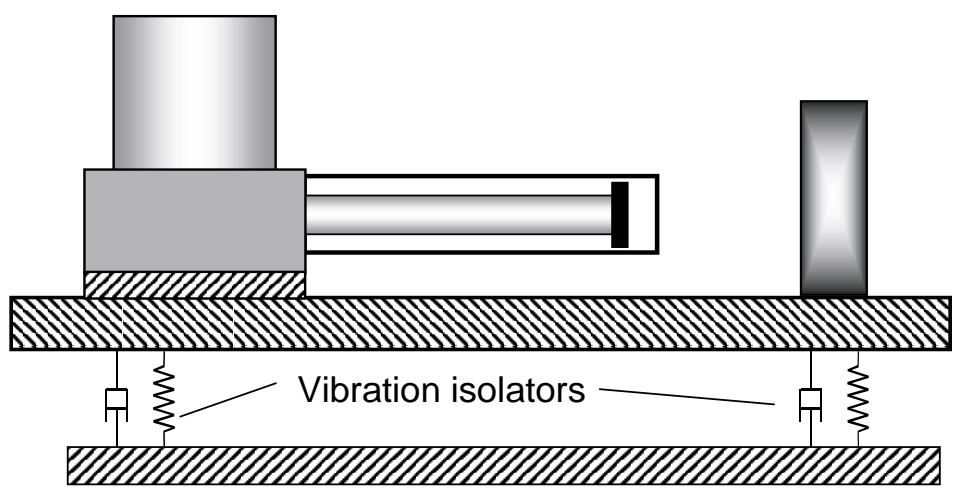

Fig. 5. Schematics of vibration isolated IR device.

As the equipment containing such a low frequency and lightly damped vibration isolator involves an exposure to the aforementioned harsh environmental conditions, which are typical for airborne applications, the problem of excessive deflections becomes a serious concern. These conditions encounter wideband random excitation (e.g. flight through turbulent flow) and high g-loads which are experienced by the airborne vehicle at take-off, climb, high-speed turn, speedup, etc. It was recently revealed that the newly designed jet fighters (e.g. Eurofighter) might develop accelerations up to $12 \mathrm{~g}$.

As a result, plenty of free "rattle space" must be allowed around the equipment, and the vibration isolators, thermal and electrical interfaces need to be of special design. Such an approach complicates the entire system and makes it both unreliable and costineffective.

An increase in the natural frequency and loss factor of an isolator allows for the close control of the above deflections. However, even where analysis of the isolation system is carried out, it is traditionally the response of the entire package that is optimised $[1,4$, 7]. Such a design eventually requires the application of a heavily (critically) damped vibration isolator. It is widespread opinion, which is supported by the leading manufacturers of vibration isolators, that only highly damped isolation materials provide the only choice for adequate protection in electronic equipment [1].

Such a concept completely misses the purpose of using isolators - to protect the sensitive internal components, and eventually calls for the application of in- 


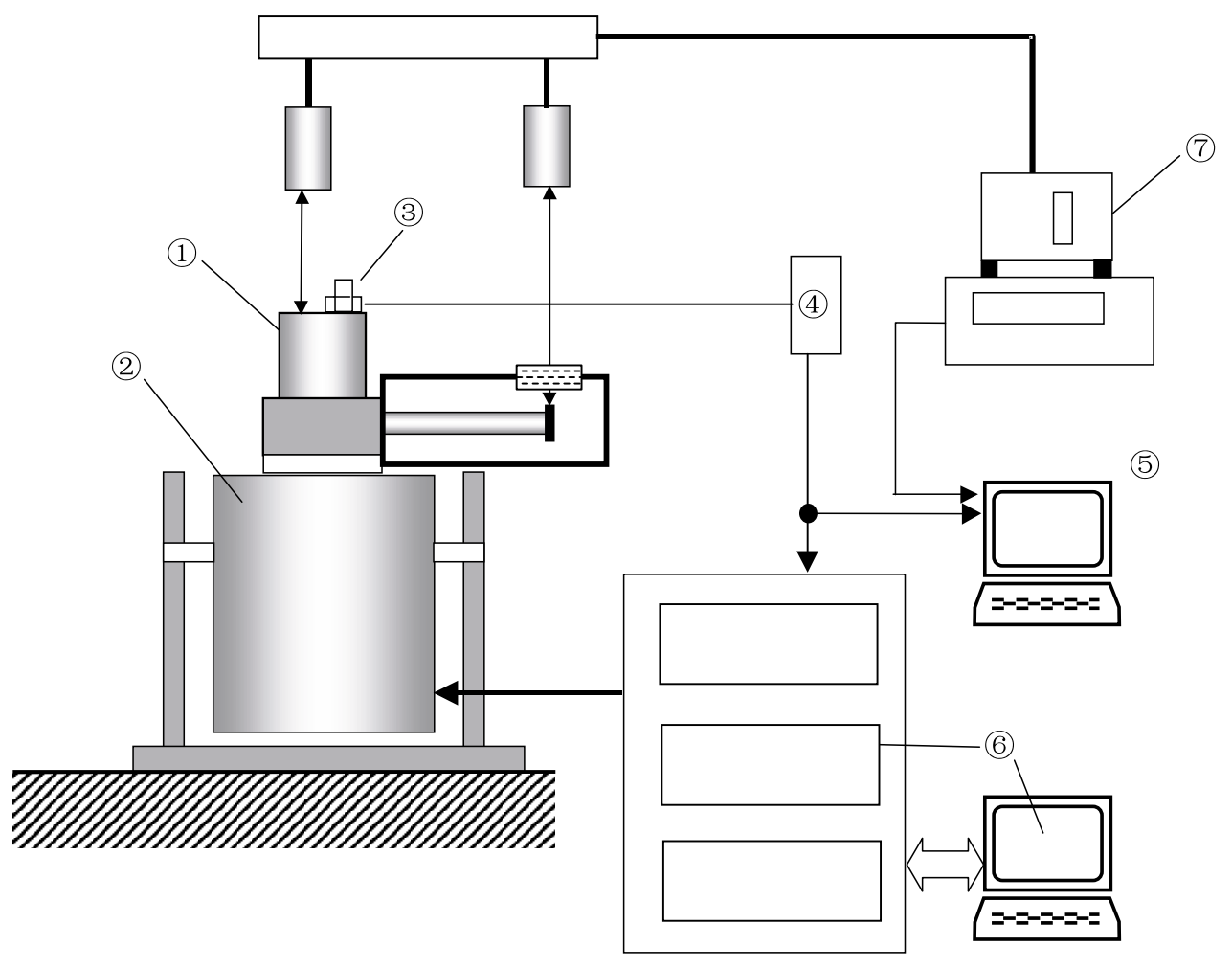

Fig. 6. Schematics of experimental rig.

adequate highly damped vibration isolators with poor vibration isolation in the high frequency range which typically contains the natural frequencies of the above critical components.

The novel approach developed in this paper is the use of vibration isolators with properties to minimise the relative dynamic response of the internal sensitive components (IR sensor relative to optic system, in this instance), subject to the restraints imposed on the peak deflections of the entire IR package. Such a design approach uses the existing equipment as the first level vibration isolation stage with respect to the internal sensitive components, and is based on the authors' ideas [8-10] in application to vibration protection of critical components in electronic equipment.

In this article, the authors develop the mathematical model of the two-degree-of-freedom (TDOF) vibration protection system and the procedure of its optimisation.

The results of numerical analysis are backed up by experiment. Under the "white noise" harsh random vibration test ( $12 \mathrm{~g} \mathrm{rms} ; 10$ to $2000 \mathrm{~Hz})$ the dynamic response of the IR sensor was reduced sixfold (from $14.4 \mu \mathrm{rms}$ to $2.3 \mu \mathrm{rms}$ ) as compared with the case of rigid mounting of the entire package. Such a level of sight-of-jitter meets the customer specification of $3 \mu \mathrm{rms}$.

The use of such an isolation system is very attractive across the industry, as no design is required in altering the sensitive internal components of existing equipment. An additional benefit is that a considerable vibration protection of the accompanying sensitive electronics and optics may be attained.

\section{Mathematical model of cold finger and IR sensor}

\subsection{Experimental study of dynamic properties of cold finger}

The experimental rig shown in Fig. 6 was created to study the dynamic properties of the system.

The cryogenic cooler (1) (RICOR, model K508A) which carries the IR sensor dummy upon the cold finger tip is mounted over the vibration exciter (2)(Ling Dymanic Systems, model V550). The control accelerometer (3)(Bruel \& Kjaer, Type 4393) is mounted on the fixture. Its signal is fed through the charge amplifier (4) (Bruel \& Kjaer, Type 2635) to the dual- 
channel vibration analyser (5) (Signal Calc Ace, Data Physics Corporation) and simultaneously to the vibration controller/power amplifier (6)(Ling Dymanic Systems, model DVC 48/PA550L). The dual-beam fibre laser vibrometer (7) (Polytec, model OFV 502) measures the velocity of the IR dummy relative to the cold finger basis. In the experiments, the vacuum envelope covered the cold finger, and measurements were taken through the transparent quartz window.

Figure 7 shows the layout of the experimental rig (a) with and (b) without the vacuum envelope.

The cryogenic cooler was subjected to the "white noise" harsh random vibration test $(12.4 \mathrm{~g}$ rms, 10 to $2000 \mathrm{~Hz}$ ). It is important to note that prior to the experiment the cryocooler was pre-cooled to simulate the actual damping properties intrinsic to the cold finger. The experimentally measured relative transfer function (transmissibility) of the cold finger is shown in Fig. 4. This curve indicates that the dynamic system under investigation behaves as a single-degree-of-freedom (SDOF) system in the frequency range encountered. From curve-fitting, the modal natural frequency $\Omega$ and loss factor $\zeta$ were estimated to be $\frac{\Omega}{2 \pi}=816 \mathrm{~Hz}$ and $\zeta=0.032$, respectively.

Figure 8 shows the actual PSD of dynamic deflection of the IR sensor relative to the base and this indicates an overall level of $14.4 \mu \mathrm{rms}$. From the spatial resolution of the typical IR sensor, only $3 \mu \mathrm{rms}$ of the overall relative deflection of the cold finger tip are allowed for smooth IR imaging.

\subsection{Mathematical model of cold finger}

As the cold finger with the mounted IR sensor behaves as SDOF system in the frequency range encountered, the mathematical description of its dynamic behaviour under the wideband random excitation may be carried out using complex universal absolute transmissibility $[3,4]$ :

$$
T(j \omega)=\frac{\Omega^{2}+2 j \omega \Omega \zeta}{\Omega^{2}-\omega^{2}+2 j \omega \Omega \zeta}
$$

and universal relative complex transmissibility

$$
\begin{aligned}
T^{r e l}(j \omega) & =T(j \omega)-1 \\
& =\frac{\omega^{2}}{\Omega^{2}-\omega^{2}+2 j \omega \Omega \zeta},
\end{aligned}
$$

where $\omega$ is the angular frequency and $j=\sqrt{-1}$ is the imaginary unity.

The PSD of the relative deflection of the IR sensor which is defined by the function $Z(t)$ may be calcu- lated $[3,4]$ in the form:

$$
S_{Z}(\omega)=\frac{1}{\omega^{4}}\left|T^{r e l}(j \omega)\right|^{2} S_{\ddot{Y}}(\omega),
$$

where $S_{\ddot{Y}}(\omega)$ is the single-sided PSD of the base acceleration, given by the function $\ddot{Y}(t)$.

The RMS of relative deflection may be derived by the integration $[3,4]$ :

$$
\sigma_{z}=\sqrt{\frac{1}{2 \pi} \int_{0}^{\infty} \frac{1}{\omega^{4}}\left|T^{r e l}(j \omega)\right|^{2} S_{\ddot{Y}}(\omega) d \omega} .
$$

By making use of the values of the natural frequency and loss factor which are obtained from curve-fitting and the numerical value of the excitation PSD $S_{\ddot{Y}}(\omega)=$ $0.08 \frac{\mathrm{g}^{2}}{\mathrm{~Hz}} @ 10-2000 \mathrm{~Hz}$, we calculate the PSD of relative deflection and the RMS value of relative deflection to be $14.9 \mu$ which is fairly close to experimentally measured value.

\section{Optimal design of vibration protection system}

\subsection{Model of vibration protection system and general relationships}

Figure 9 shows the model of a TDOF vibration protection system, where the primary sub-system has the modal natural frequency $\Omega_{1}$ and loss factor $\zeta_{1}$ and represents the vibration isolated IR package. The secondary sub-system has the modal natural frequency $\Omega$ and loss factor $\zeta$ and represents the cold finger with the mounted IR sensor. The base vibration is given by the function $Y(t)$. The absolute deflection of the primary and secondary subsystems are $X_{1}(t)$ and $X(t)$. The relative deflection of the primary subsystem to the base is $Z_{1}(t)=X_{1}(t)-Y(t)$, and relative deflection of the secondary subsystem to the primary system is $Z(t)=X_{2}(t)-X_{1}(t)$.

It is important to note that the mass of the secondary subsystem is negligibly small as compared to the primary subsystem. In this particular case the mass of the sensor and effective mass of the cold finger was approximately 200 times lighter than the entire system. Therefore, the dynamic response of the primary system may be considered to be independent of the secondary subsystem. It may also be thought of as a vibration input to the secondary subsystem.

The absolute and relative complex transmissibilities of the primary subsystem are derived similarly to (1) and (2) in the form: 


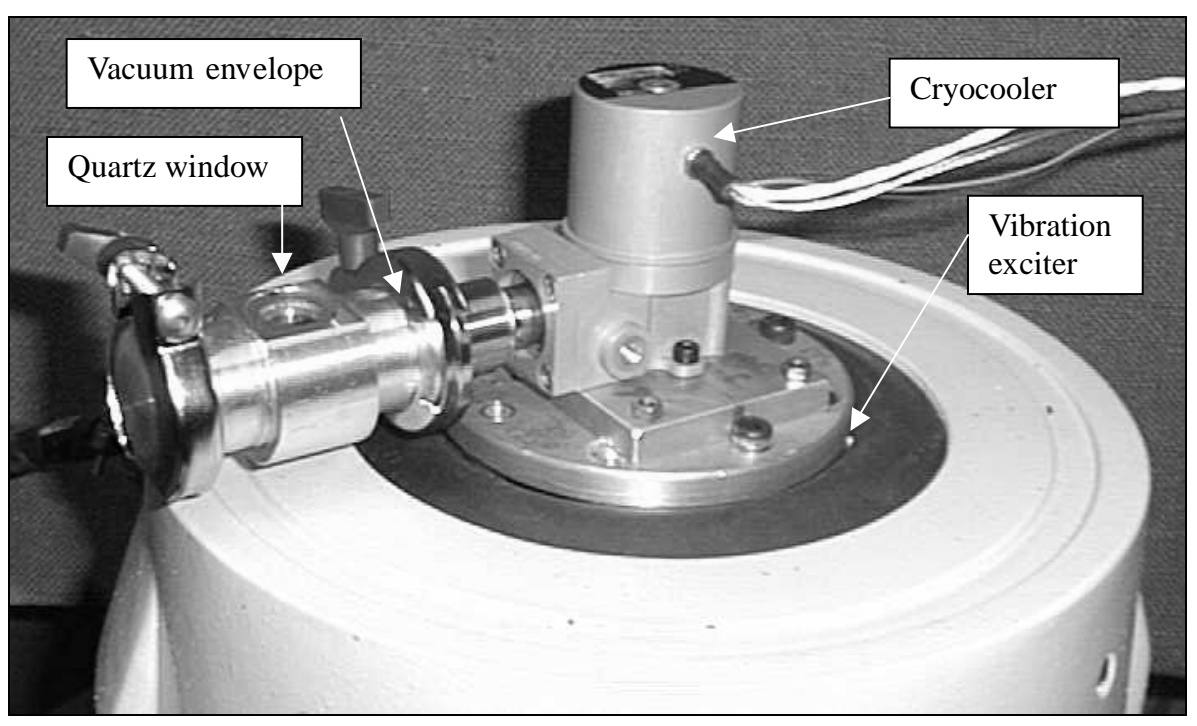

(a)

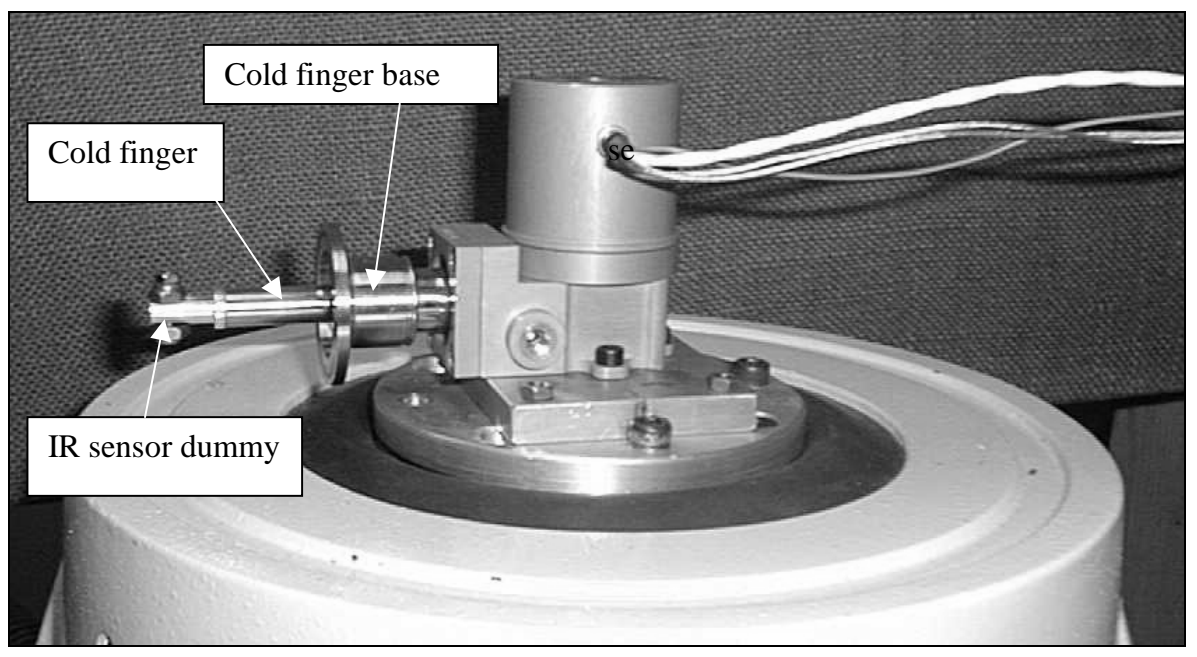

(b)

Fig. 7. Layout of experimental rig.

$$
\begin{aligned}
T_{1}(j \omega) & =\frac{\Omega_{1}^{2}+2 j \omega \Omega_{1} \zeta_{1}}{\Omega_{1}^{2}-\omega^{2}+2 j \omega \Omega_{1} \zeta_{1}} . \\
T_{1}^{r e l}(j \omega) & =T_{1}(j \omega)-1 \\
& =\frac{\omega^{2}}{\Omega_{1}^{2}-\omega^{2}+2 j \omega \Omega_{1} \zeta_{1}} .
\end{aligned}
$$

The PSD and RMS of absolute acceleration of the primary subsystem are calculated in the form [3,4]:

$$
S_{\ddot{X}_{1}}(\omega)=\left|T_{1}(j \omega)\right|^{2} S_{\ddot{Y}}(\omega),
$$

$$
\sigma_{\ddot{X}_{1}}=\sqrt{\frac{1}{2 \pi} \int_{0}^{\infty} S_{\ddot{X}_{1}}(\omega) d \omega} .
$$

Similarly, the PSD and RMS of relative deflection of the primary subsystem are obtained in the form:

$$
S_{Z_{1}}(\omega)=\frac{1}{\omega^{4}}\left|T_{1}^{r e l}(j \omega)\right|^{2} S_{\ddot{Y}}(\omega),
$$

$$
\sigma_{Z_{1}}=\sqrt{\frac{1}{2 \pi} \int_{0}^{\infty} S_{Z_{1}}(\omega) d \omega}
$$




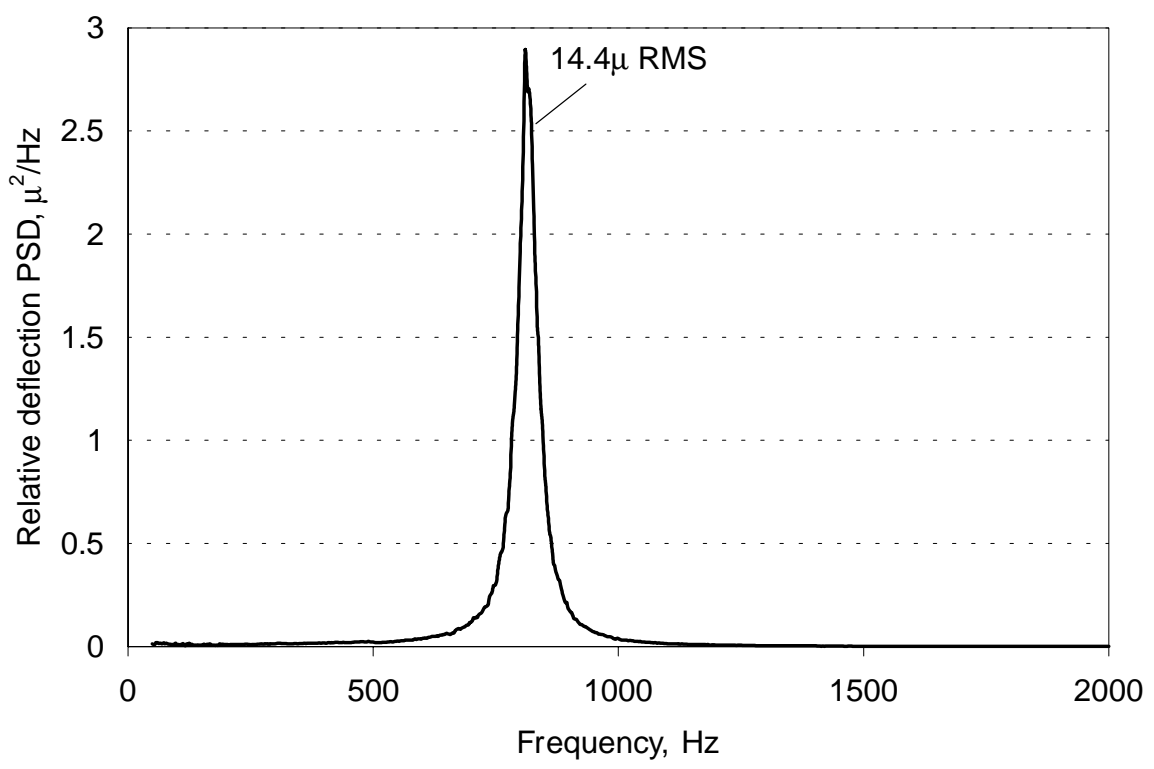

Fig. 8. Experimentally measured dynamic response of cold finger.

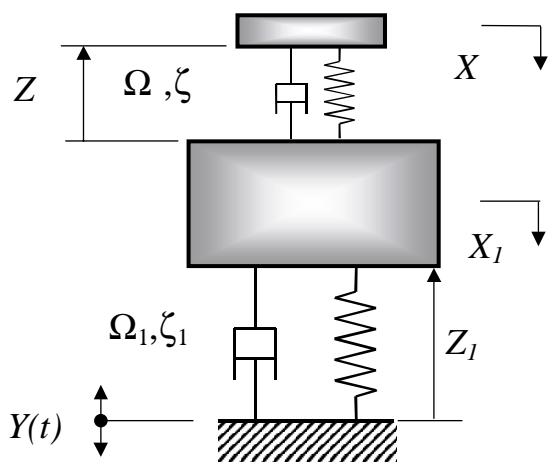

Fig. 9. Model of vibration protection system.

In applying the $3 \sigma$ rule [7], which provides for the instantaneous level of normally distributed deflection to be less then $3 \sigma$ with a probability of $99.73 \%$, and accounting for the additional quasi-static relative deflection due to the g-loading, the total peak relative deflection may be derived by means of the expression:

$$
Z_{1}^{\text {peak }}=3 \sigma_{Z_{1}}+\frac{G}{\Omega_{1}^{2}},
$$

where $G$ is the specified level of g-loading.

The vibration of the primary subsystem may be thought of as the excitation to the secondary subsystem, therefore the PSD and RMS of relative deflection may be calculated as follows:

$$
S_{Z}(\omega)=\frac{1}{\omega^{4}}\left|T^{r e l}(j \omega)\right|^{2} S_{\ddot{X}_{1}}(\omega)
$$

and

$$
\sigma_{Z}=\sqrt{\frac{1}{2 \pi} \int_{0}^{\infty} S_{Z}(\omega) d \omega}
$$

\subsection{Statement and solution to optimal problem}

As stated above, even large motion of the entire IR package, which does not give rise to the excessive displacement of an IR detector relative to the optic system, does not affect the quality of imaging of the remote targets. Therefore, the major objective of optimal design of such a vibration protection system is the minimisation of the relative deflection of the secondary subsystem to the primary one, subject to constraints of the limited peak deflections of the primary subsystem to the base. These constraints are typically imposed by the 


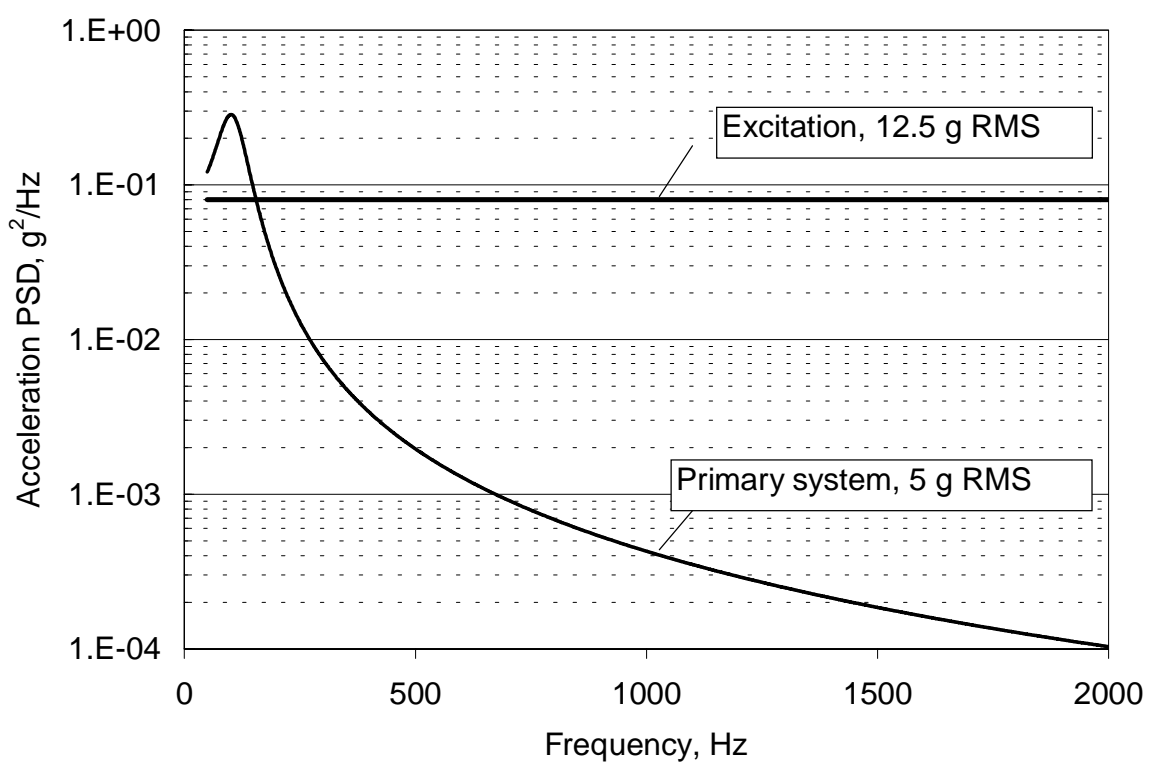

Fig. 10. Excitation and dynamic response of the optimised vibration isolator.

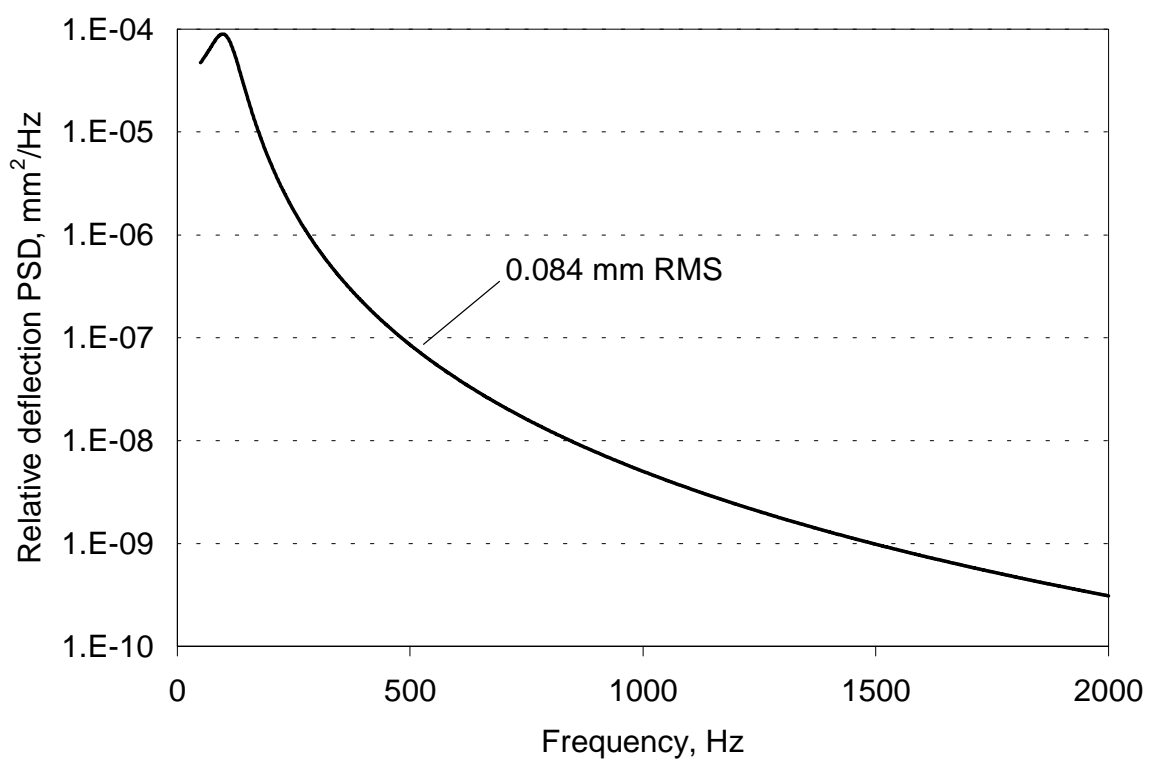

Fig. 11. Dynamic deflection of optimised vibration isolator.

design of the electro-optic device enclosure, electrical harness and thermal interfaces, etc.

Mathematically this may be expressed in the form:

$$
\sigma_{z} \rightarrow \min ; \quad Z_{1}^{\text {peak }} \leqslant \Delta
$$

where $\Delta$ is the allowable peak deflection of the primary subsystem.

Since the natural frequency and loss factor of the cold finger are not supposed to be altered, we consider that $\frac{\Omega_{2}}{2 \pi}=816 \mathrm{~Hz}$ and $\zeta_{2}=0.032$ (as obtained above from the curve-fitting). The PSD of the excitation is $S_{\ddot{Y}}(\omega)=0.08 \frac{\mathrm{g}^{2}}{\mathrm{~Hz}}$ in the frequency range $10-2000 \mathrm{~Hz}$. The maximum level of g-loading is $12 \mathrm{~g}$. The allowable peak deflection of the electro-optic package is $\Delta=$ $0.5 \mathrm{~mm}$.

The remaining two variables, namely, the natural frequency and loss factor of the primary subsystem $\Omega_{1}$ and $\zeta_{1}$, may be manipulated to meet the conditions of 


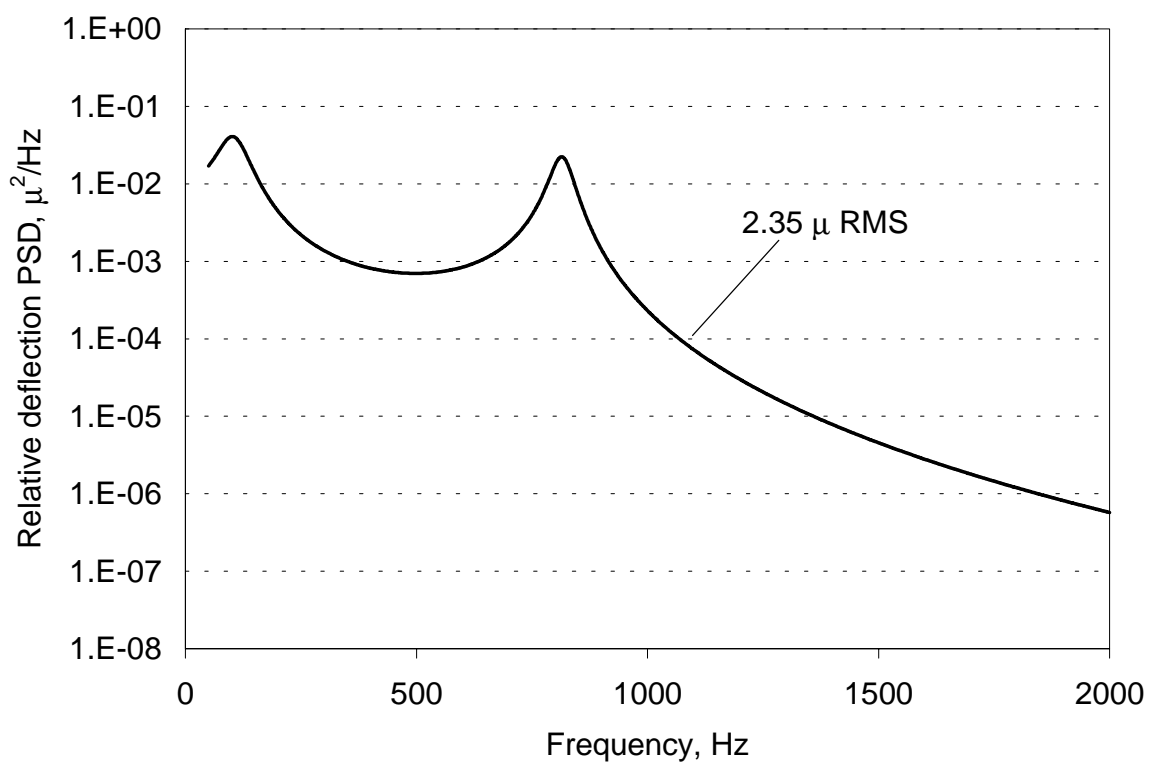

Fig. 12. Relative deflection of the vibration protected cold finger.

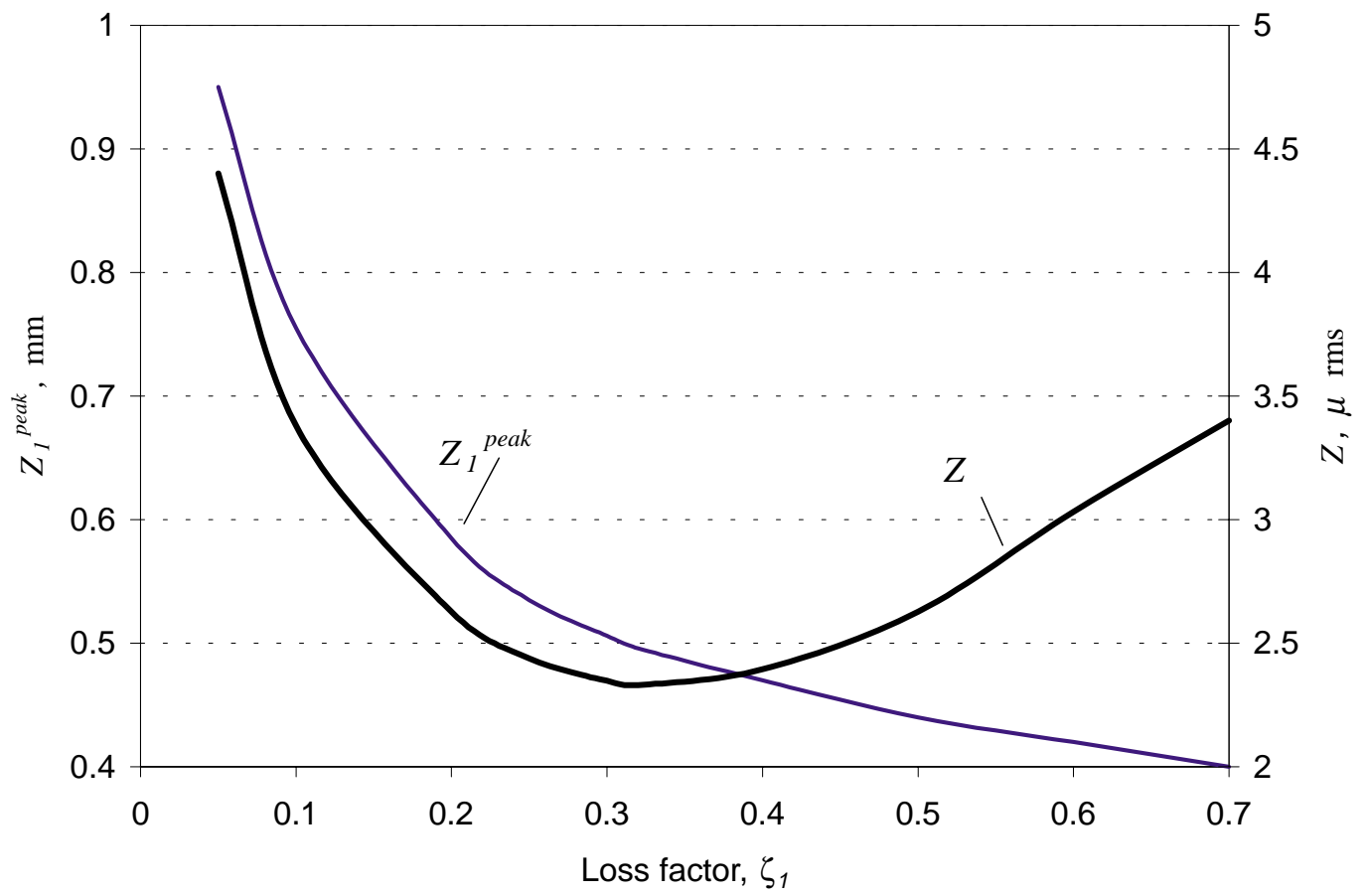

Fig. 13. Analysis of sensitivity.

(14).

The expressions (1), (2), (5), (7)-(14) were involved in the design of an appropriate MSExcel worksheet. The procedure of numerical optimisation relies on the application of the standard Solver add-in procedure.

As a result of the solution to the optimal problem
(14), the "optimal" primary vibration isolator was determined to have the parameters:

$$
\frac{\Omega_{1}^{o p t}}{2 \pi}=110 \mathrm{~Hz}, \quad \zeta_{1}^{o p t}=0.32 .
$$

Under the condition that the primary vibration isola- 


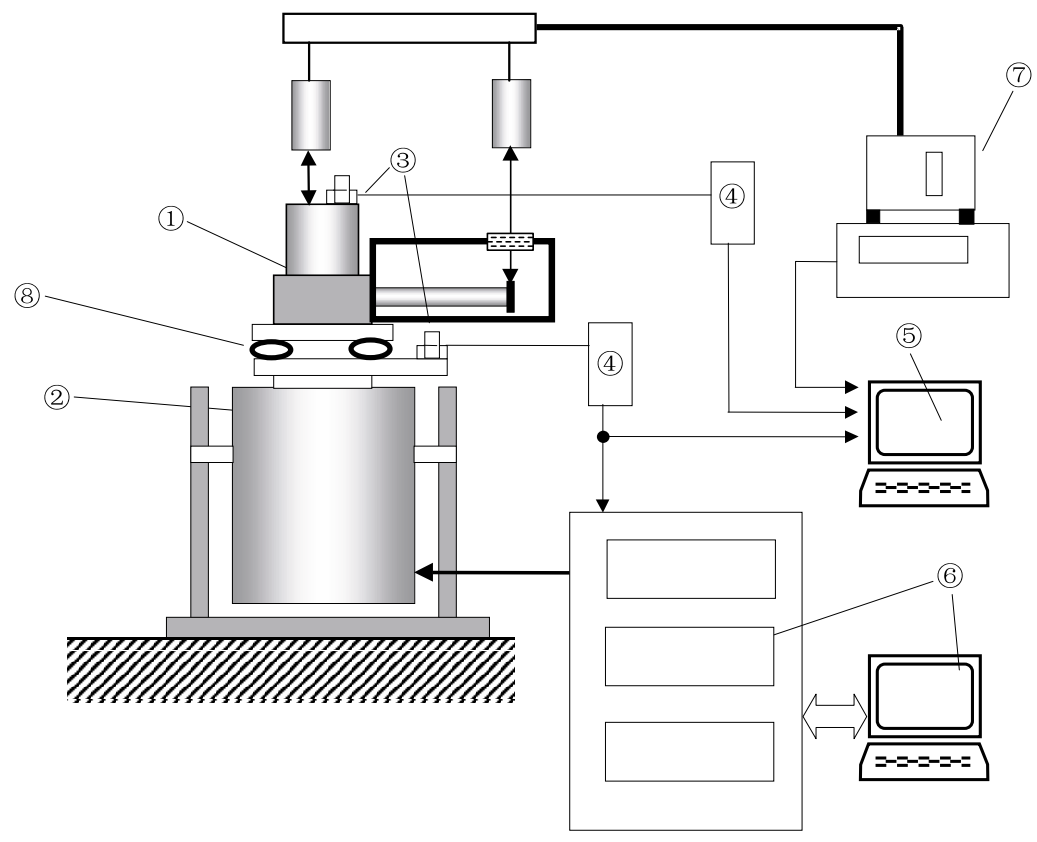

Fig. 14. Experimental rig.

tor is chosen in accordance with (15), the overall level of vibration experienced by the electro-optic package is $\sigma_{\ddot{X}_{1}}=5 \mathrm{~g}$ (attenuation factor 2.9, as compared with the base overall vibration level). The peak deflection of the entire package relative to the base is $z_{1}^{\text {peak }}=$ $0.5 \mathrm{~mm}$, while the dynamic component is $0.253 \mathrm{~mm}$ and quasi-static component is $0.247 \mathrm{~mm}$.

At the same time the overall level of relative dynamic deflection of the IR sensor to the cold finger base is $\sigma_{Z}=2.35 \mu \mathrm{rms}$ which indicates a sixfold attenuation as compared to the case of rigid mounting of the entire IR package.

Figure 10 compares the PSD of excitation acceleration and that of the vibration isolated package. Figure 11 shows the PSD of the relative deflection of the electro-optic package to the base showing the overall level of $0.084 \mathrm{~mm}$ rms. Figure 12 shows the PSD of relative deflection of the IR sensor to the cold finger base showing the mentioned overall level of $2.35 \mu \mathrm{rms}$ (compare with Fig. 8).

\subsection{Analysis of sensitivity}

Since the vibration protection system relies on an optimised vibration isolator, it is important to determine the sensitivity of the dynamic response of the IR sensor to the properties of the primary isolator.

Figure 13 shows, for example, the variation of the dynamic responses of both primary and secondary sub- systems in response to the variation in the loss factor of the primary isolator the natural frequency of which remains constant, $\frac{\Omega_{1}}{2 \pi}=110 \mathrm{~Hz}$. An analysis of this Figure indicates the low sensitivity of the dynamic responses of both primary and secondary subsystems to relatively large deviations of the loss factor from its optimal value.

\subsection{Choice of vibration isolator}

In the experiments we used standard, commercially available Shock Tech ${ }^{1}$ Cable Mounts (see also Enidine, ${ }^{2}$ Barry Controls, ${ }^{3}$ Aeroflex International [12]) providing for the desired loss factor and natural frequency. Such cable mounts are of all-metal design, constructed out of stainless steel cable and aluminium bars, and especially intended to withstand the severe environmental conditions while demonstrating no outgassing and ageing, long fatigue life and persistence of parameters in a wider temperature range, as compared with the polymer isolators. The wire rope cables in these isolators are inherently damped through internal wire flexure hysteresis, thus providing for the loss factor to be in the range of $30 \%$ (treated cable) [12].

\footnotetext{
${ }^{1}$ See http://www.shocktech.com/products.htm.

${ }^{2}$ See http://www.enidine.com/Wire\%20Rope/WireRope_ 1.html\# WireRopePDF.

${ }^{3}$ See http://www.bpg-inc.com/barry.htm.
} 


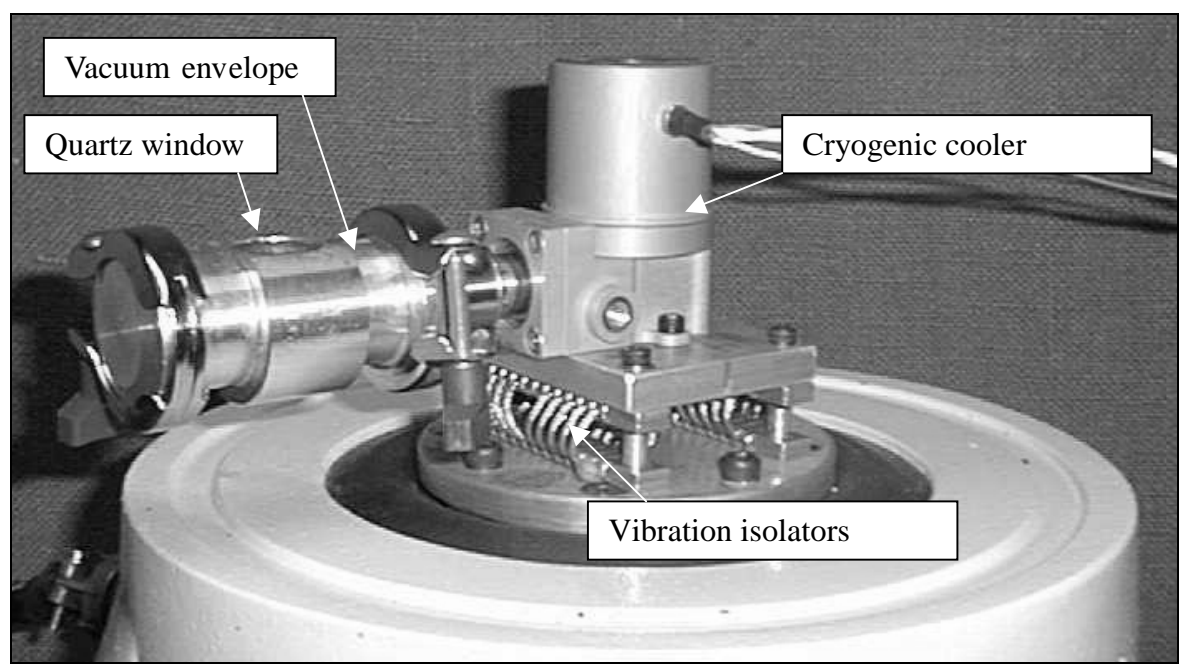

(a)

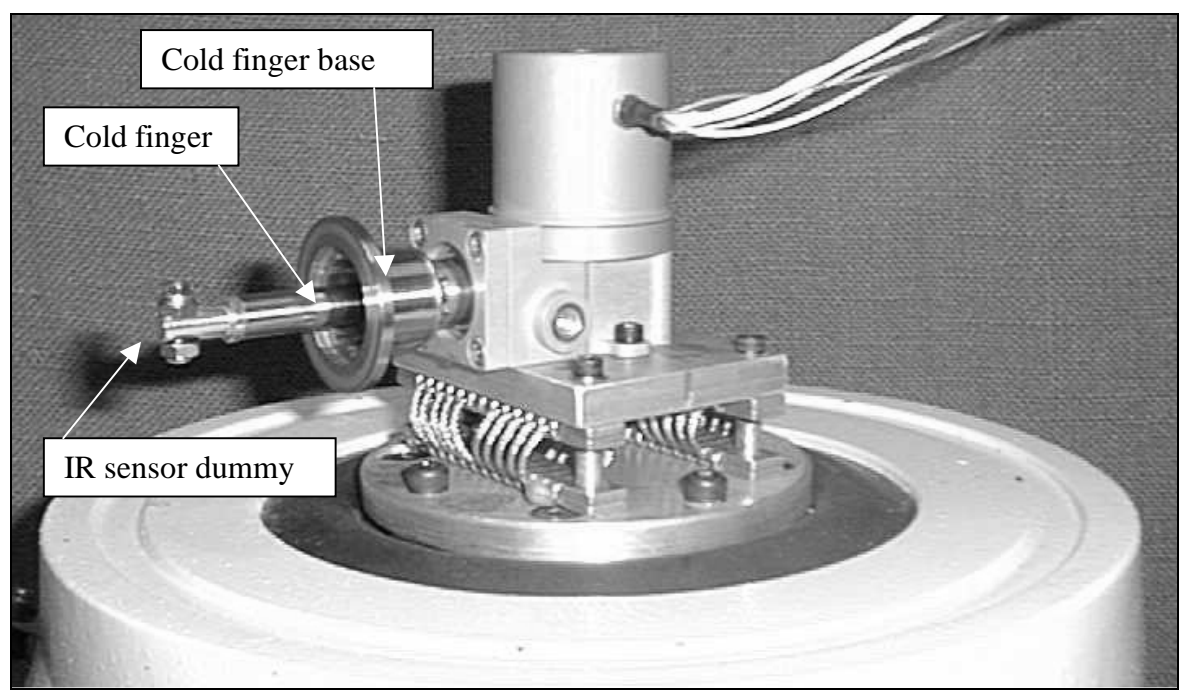

(b)

Fig. 15. Layout of experimental rig.

Since the quality of the vibration protection system depends strongly on the parameters of the primary suspension, this feature is, probably, the most critical for the choice of a proper vibration isolator.

\section{Experiment}

\subsection{Test rig}

Figure 14 shows the schematics of the experimental rig. In general, the notations are similar to those in
Fig. 6. The cryogenic cooler is suspended from the vibration exciter table by means of two Shock Tech Cable Mounts (8).

Additional accelerometer and charge amplifiers are used for measuring the dynamic response of the cryogenic cooler, which, in this case, is definitely different from the motion of the vibration exciter. During the experiments the vacuum envelope was mounted to cover the cold finger and measurements were taken through the transparent window.

Figure 15 shows the layout of the experimental rig (a) with and (b) without the vacuum envelope. 


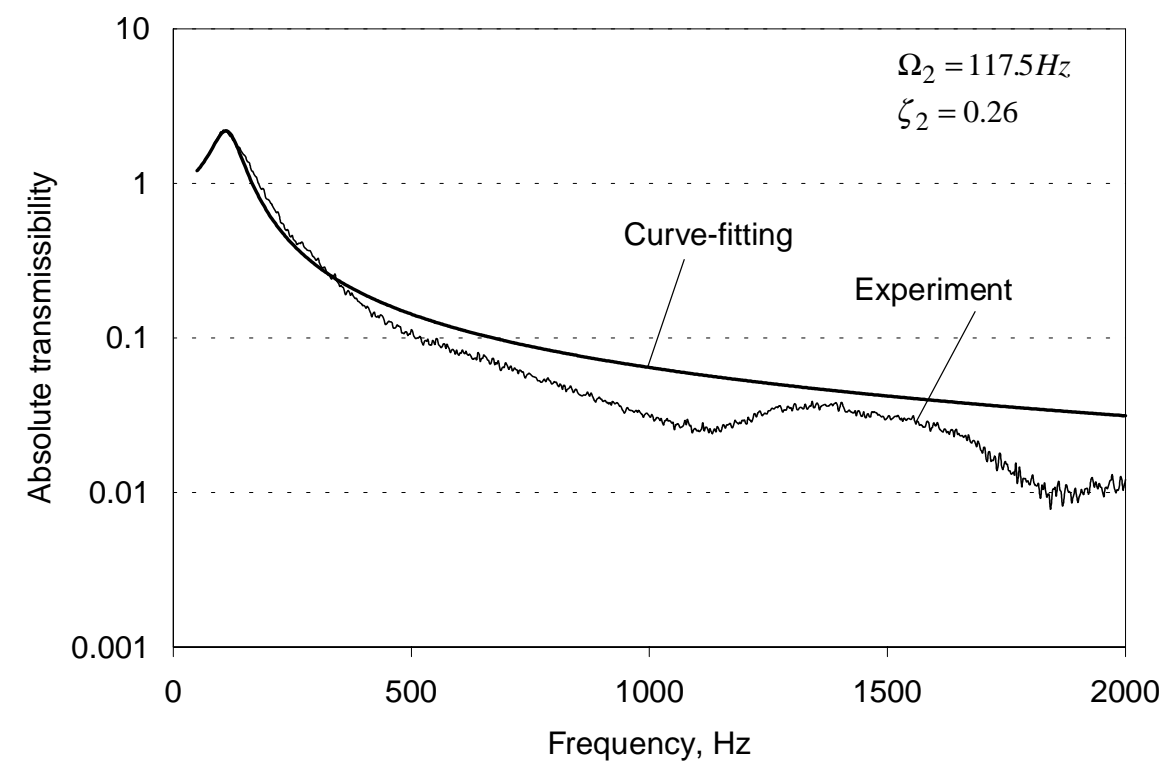

Fig. 16. Experimentally measured absolute transmissibility of the primary vibration isolator and estimation of its modal parameters by curve-fitting.

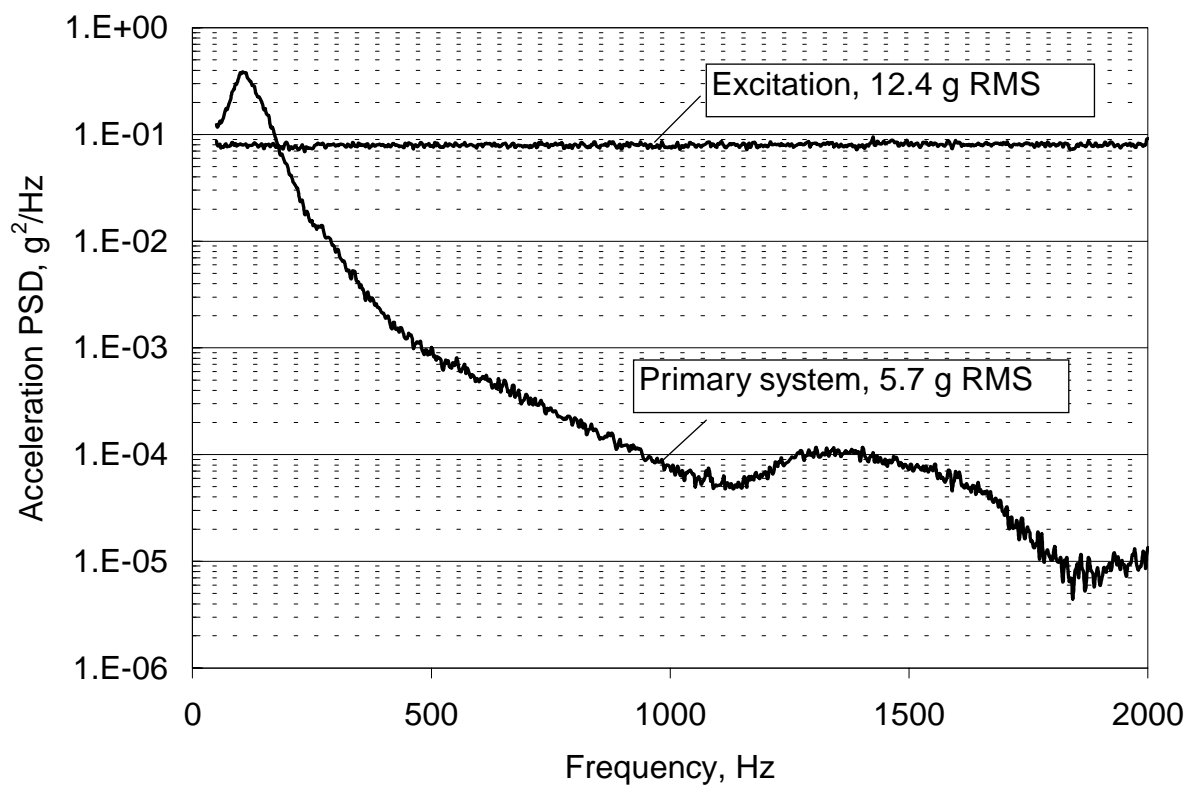

Fig. 17. Experimentally measured excitation and dynamic response of the primary vibration isolator.

\subsection{Results of measurements}

Figure 16 shows the experimentally measured absolute transmissibility of the primary subsystem (label Experiment). From curve-fitting, the natural frequency and loss factor are estimated as $117.5 \mathrm{~Hz}$ and 0.26 , respectively. These values are only slightly different from the optimal values (15).
Figure 17 compares the PSD of excitation and acceleration of the primary subsystem. The obtained results are in a close agreement with analytical prediction (see Fig. 10).

Figure 18 shows the experimentally measured PSD of the relative deflection of the primary subsystem showing the $0.24 \mathrm{~mm}$ peak deflection (the $3 \sigma$ rule was applied to the $0.08 \mathrm{~mm}$ rms value). These experimental 


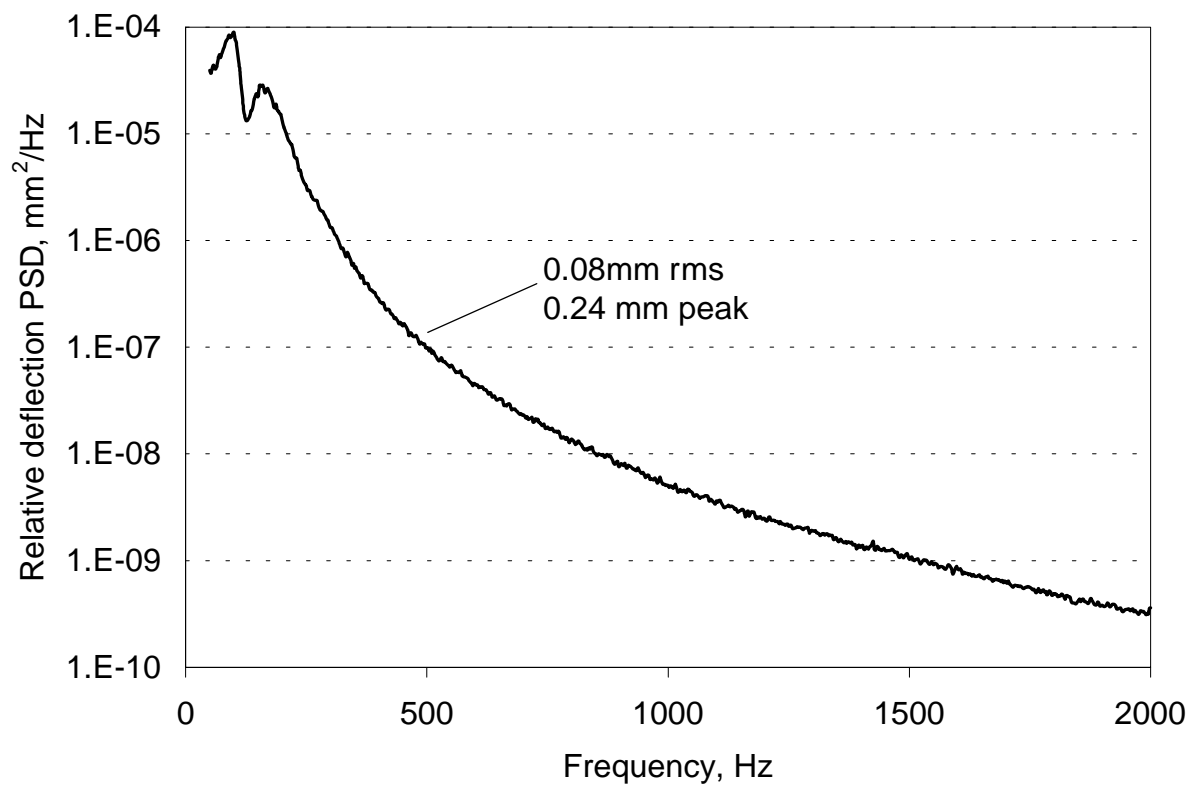

Fig. 18. Experimentally measured dynamic response of the primary vibration isolator.

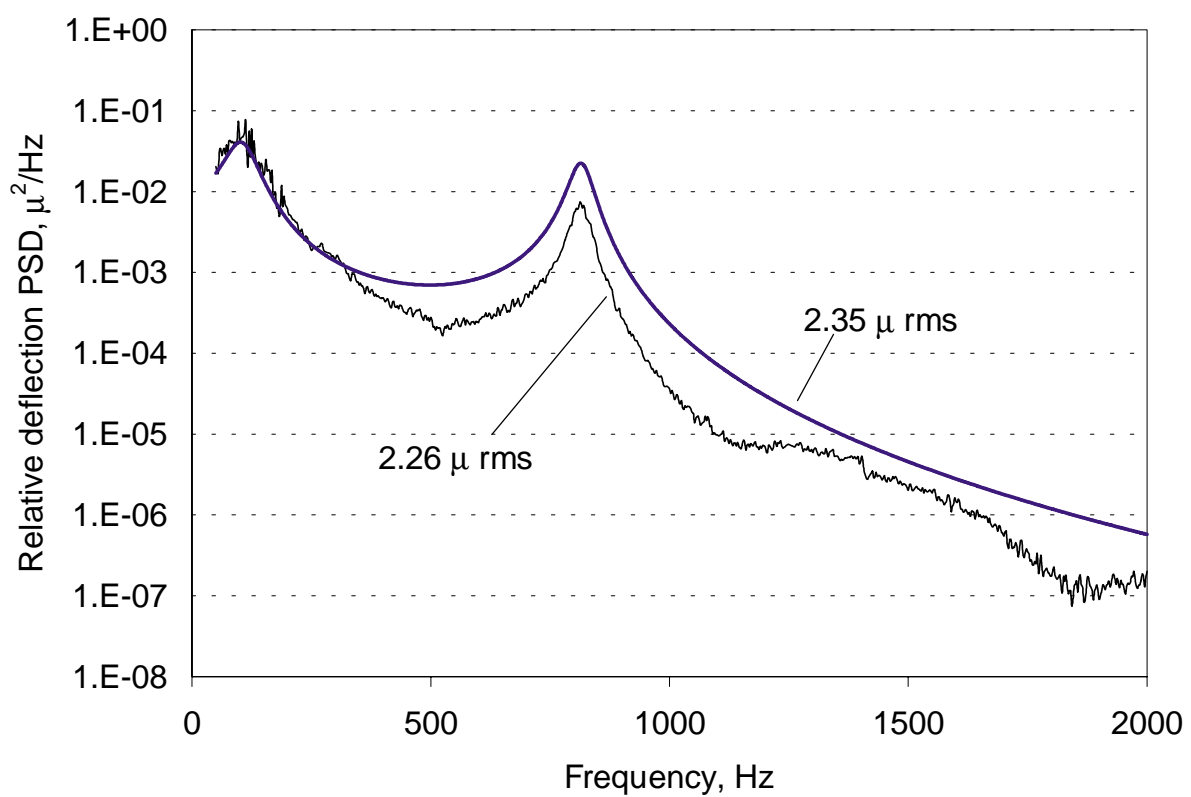

Fig. 19. Analytically predicted (2.35 $\mu \mathrm{rms})$ and experimentally measured (2.26 $\mu \mathrm{rms})$ dynamic response of cold-finger.

results are also in good agreement with the analytical prediction in Fig. 11.

Finally, Fig. 19 shows the experimentally measured PSD of the relative deflection of the cold finger tip which indicates the overall level of $2.26 \mu \mathrm{rms}$ (compare this with $2.35 \mu \mathrm{rms}$ from the analytical prediction). The closeness of the obtained results is evident.

\section{Conclusions}

In this article the authors suggest that a relatively heavy electro-optic device should be used as the firstlevel vibration isolation stage relative to the sensitive internal components of the IDCA package. For this purpose they developed an approach to the optimal design of the vibration isolators with properties chosen to 
minimise the response of the IR sensor relative to the rest of the optic system, subjected to strict constraints on the allowable sway space of the entire electro-optic package.

It was predicted analytically and confirmed experimentally, that the proposed vibration protection system would be capable of a sixfold reduction in the relative dynamic displacement of the IR sensor as compared with the case of its rigid mounting.

The proposed approach may provide benefit across a wide range of applications. The vibration protection arrangement described may be applied widely when the high-quality rugged and inexpensive dynamic protection of sensitive IR equipment is required. The use of such an isolation system is very attractive across the industry, as no design is required in altering the sensitive internal components of existing equipment. Additionally, considerable vibration protection of the accompanying sensitive electronics and optics of the IDCA package may be achieved.

\section{References}

[1] E-A-R Specialty Composites, Protecting Electronic Equipment from Shock and Vibration, Machine Design, September
1995, pp. 60-61.

[2] L.A. Coleman, Infrared Sensors: The Eyes of the Digital Battlefield, Military \& Aerospace Electronics (July 1994), 2931.

[3] S.H. Crandall and W.D. Mark, Random vibration in mechanical systems, Academic Press, NY, 1963.

[4] C.M. Harris, Shock and vibration handbook, (4th ed.), Publisher New York, London, McGraw-Hill, 1996.

[5] Y. Ikuta, Y. Suzuki, K. Kanao and N. Watanabe, Development of a long-life Stirling cryocooler, Proceedings of the 11th International Cryocooler Conference, Keystone, Colorado, 2000.

[6] J.L. Miller, Principles of Infrared Thechnology, Van Nostrand Reinhold, 1994.

[7] J.L. Sloan, Design and Packaging of Electronic Equipment, Van Nostrand Reinhold Company, 1985.

[8] A.M. Veprik, Vibration protection of the electro-optic equipment, Invited lectures on International Conference "ElectroPack' 95", Israel, 1995.

[9] A.M. Veprik and V.I. Babitsky, Optimal external mounting for vibration protection of lightweight internal components of electronic equipment, Proceedings of the 6th International Congress on Sound and Vibration, Technical University of Denmark, Lyngby, July 1999.

[10] A.M. Veprik and V.I. Babitsky, Vibration protection of sensitive electronic equipment from harsh harmonic vibration, Journal of Sound and Vibration 238(1) (2000), 19-30.

[11] G. Walker, Cryogenic coolers, Part 2 - Applications, Plenum Press, New York, 1983.

[12] E. Jasson, COTS Journal, Shock and Vibration Isolators for COTS Equipment, http://www.rtcgroup.com/cotsjournal/ cotsjulyaugustp27.html, July-August 1999. 

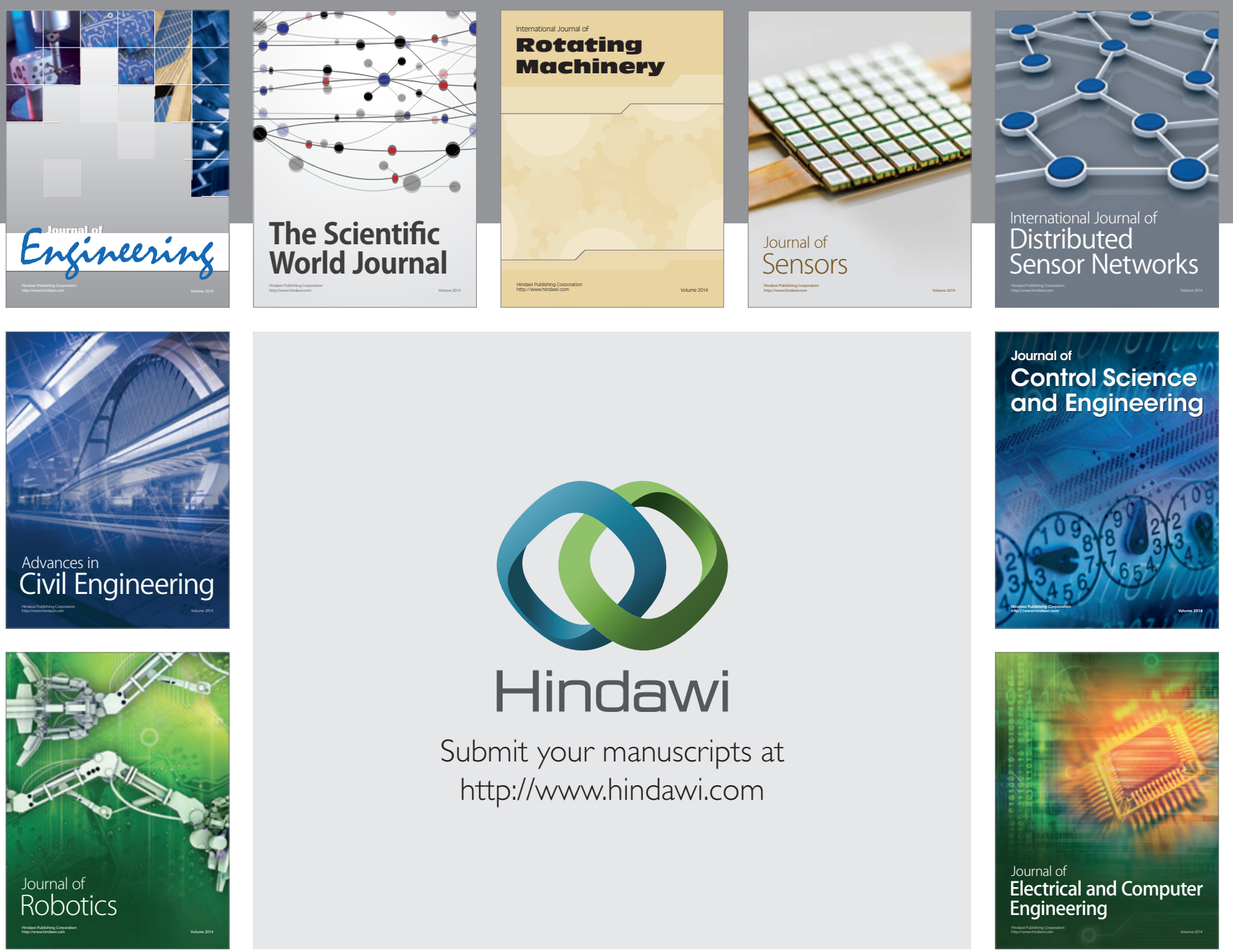

Submit your manuscripts at

http://www.hindawi.com
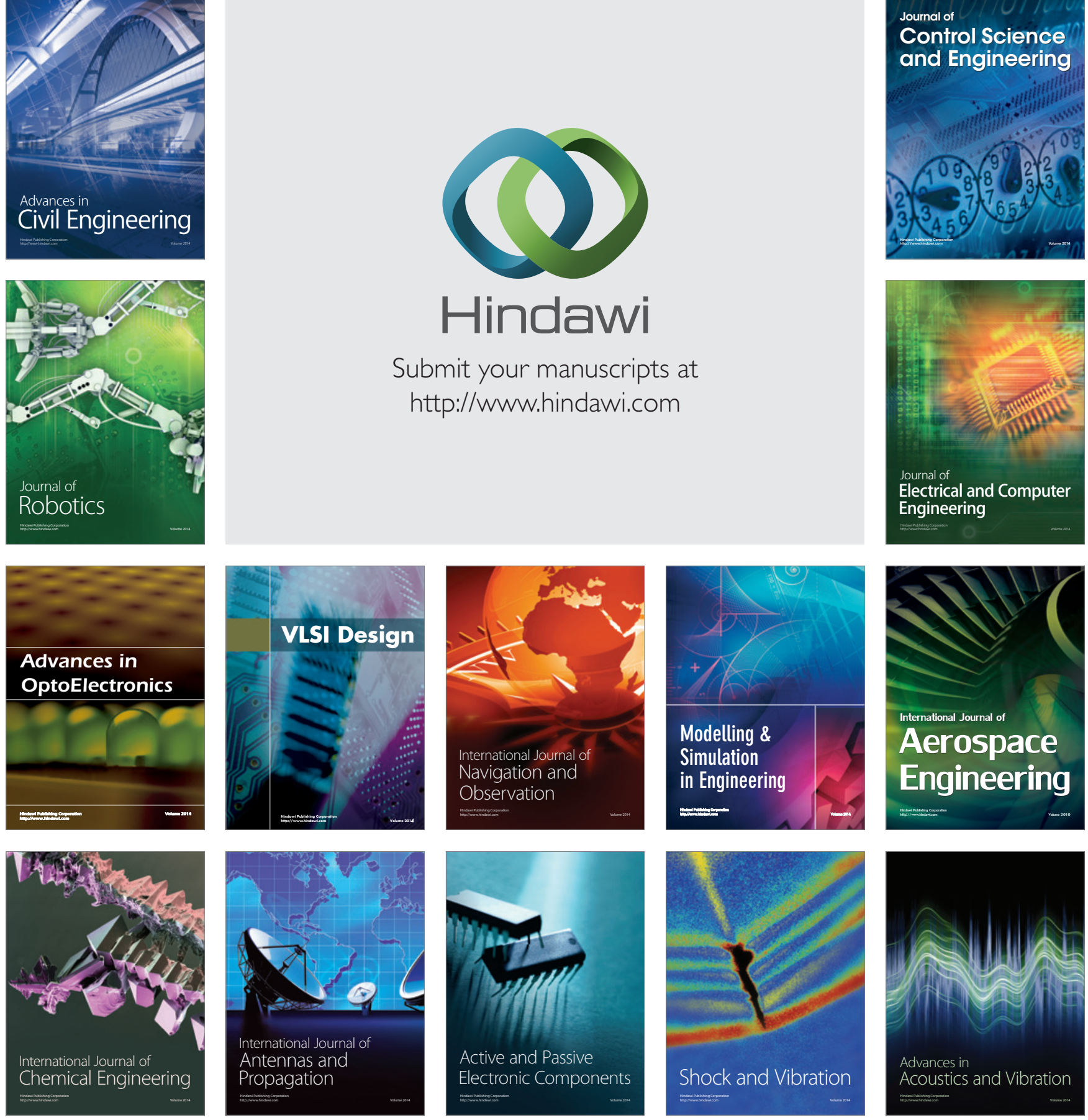